\title{
Effect of Graded Early Mobilization on Psychomotor Status and Length of Intensive Care Unit Stay in Mechanically Ventilated Patients
}

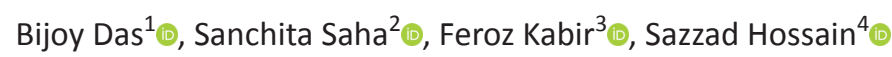

\begin{abstract}
Introduction: The main purpose of this study was to evaluate the effectiveness of graded early mobilization on psychomotor status and duration of ICU stay of patients with mechanical ventilation.

Materials and methods

Design: Quasi-experimental study.

Setting: BRB Hospitals Limited, Dhaka, Bangladesh. A reputed 500-beddedgeneral hospital with 30 ICU beds.

Participants: A total of 30 patients were selected as participants in the study from the hospital. 15 patients were included in the ICU treatment group and the remaining 15 were in the intervention group using the purposive sampling method.

Scales used: Functional independence measure (FIM) scale and 7 point generalized anxiety depression (GAD-7) scale.

Intervention: Graded early mobilization was provided as an intervention to all participants of the intervention group by a professionally qualified ICU physiotherapist for 10 sessions. Most of the patients received multiple sessions of intervention within a day.

Results: In the control group mean FIM score was 17.40 (SD \pm 4.88 ), and in the intervention group mean score was $65.70(S D \pm 12.18)$. The mean difference was statistically significant in the ' $t$ ' test ( $p$-value $>0.001$ ). In the control group, the mean GAD-7 score was 19.50 (SD \pm 2.71 ), and in the intervention group the mean GAD-7 score was 7.5 (SD \pm 2.59 ). The mean difference was statistically significant in the' $t$ 'test. ( $p$-value $>0.001)$. The mean length of ICU stay in the control group was 5.60 (SD \pm 1.07 ) and in the intervention group it was 3.10 (SD \pm 0.56 ). The mean difference was statistically significant in the ' $t$ ' test ( $p$-value $>0.001$ ).

Conclusion: This research showed that graded early mobilization was highly effective to improve the motor and psychological status of mechanically ventilated patients and reduce their length of ICU stay.

Keywords: Functional status, Graded early mobilization, Intensive care unit, Mechanical ventilation, Psychological status, Physiotherapy.

Indian Journal of Critical Care Medicine (2021): 10.5005/jp-journals-10071-23789
\end{abstract}

\section{BACKGROUND}

Mechanical ventilation (MV) is a life-support therapy that improves anoxia, carbon dioxide retention, and acid-base equilibrium. Due to the focus on stabilization of life-threatening pathophysiologic changes, little attention has been paid to neuromuscular and longterm cognitive function in such critically ill patients. ${ }^{1-9}$

In most of the intensive care units (ICUs), bed rest is considered as the routine standard of care which leads to immobility, deconditioning, and weakness. Critically ill patients in the ICU commonly receive less than $60 \%$ of their nutritional intake during their ICU stay leading to further malnutrition. The presence of muscle weakness is associated with the duration of mechanical ventilation and length of ICU stay. Muscle strength decreases to $20 \%$ within one week of immobilization with an additional decrease of $20 \%$ in each subsequent week. ${ }^{10,11}$

\section{Materials and Methods}

\section{Design}

Considering the availability of participants in the ICU that met the selection criteria and given time frame by the research Ethics committee for data collection quasi-experimental design was selected as the research design.
${ }^{1}$ Department of Physiotherapy, BRB Hospitals Limited, Dhaka, Bangladesh

${ }^{2}$ Department of Physiotherapy, Centre for the Rehabilitation of the Paralysed (CRP), Mirpur, Dhaka, Bangladesh

${ }^{3}$ Department of Physiotherapy and Rehabilitation, Jashore University of Science and Technology, Jashore, Bangladesh

${ }^{4}$ Department of Intensive Care Unit, BRB Hospitals Limited, Dhaka, Bangladesh

Corresponding Author: Bijoy Das, Department of Physiotherapy, BRB Hospitals Limited, Dhaka, Bangladesh, Phone: +880 1818506528, e-mail: dr.bjoy@gmail.com

How to cite this article: Das B, Saha S, Kabir F, Hossain S. Effect of Graded Early Mobilization on Psychomotor Status and Length of Intensive Care Unit Stay in Mechanically Ventilated Patients. Indian J Crit Care Med 2021;25(4):416-420.

Source of support: Nil

Conflict of interest: None

\section{Setting}

BRB Hospitals Limited, Dhaka, Bangladesh. A reputed 500-bedded general hospital with 30 ICU beds. 


\section{Participants}

A total of 50 patients were selected as participants in the study from the hospital. Twenty-five patients were included in the ICU treatment group and the remaining 25 were in the intervention group using the purposive sampling method.

\section{Intervention}

Graded early mobilization was provided as an intervention to all participants of the intervention group by a professionally qualified ICU physiotherapist.

\section{Process of Application of Intervention}

Graded early mobilization was provided as an intervention to all participants of the intervention group by a professionally qualified ICU physiotherapist for 10 sessions to each participant. Most of the patients received multiple sessions ( $2-3$ sessions) of intervention within a day.

Another senior physiotherapist was assigned as a data collector for both control and intervention groups to reduce bias and ensure the blindness of the experiment. Written consent was obtained from legal guardians ofall patients initially when patients were in ICU. But patient concerns were also addressed and they were explained all the areas of the study when they were capable to talk.

\section{Scales Used}

Functional independence measure (FIM) scale and7 point generalized anxiety depression (GAD-7) scale.

\section{Interventions}

\section{Graded Early Mobilization Protocol}

According to this protocol, graded early mobility can be defined as beginning the mobility program when the patient is minimally able to participate in the therapy, has stable hemodynamic status, and is receiving acceptable levels of oxygen. The criteria for mobilization were heart rate less than $110 / \mathrm{min}$ at rest, mean arterial blood pressure between $60 \mathrm{~mm} \mathrm{Hg}$ and $110 \mathrm{~mm} \mathrm{Hg}$, the fraction of inspired oxygen less than 0.6 , and oxygen saturation on activity greater than $88 \%$. The vitals of the patient were to be assessed before, during, and after any mobility intervention. ${ }^{12-19}$

The protocol was modified due to practical concerns and was divided into four phases.

\section{Phase 1}

This included patients who were critically ill with multiple medical problems, had limited activity tolerance and were unable to walk. The goal of phase 1 was to make the patient sit at the edge of the bed unsupported or with minimal assistance and initiate standing with manual assistance and walker support. General criteria for progressing to the next phase were that the patient followed commands, had stable hemodynamic and acceptable oxygenation, and was able to stand with a walker. ${ }^{20-26}$

\section{Phase 2}

This phase included patients in the acute/subacute phase with multiple medical problems, in a stable condition, and able to participate better in the activities. The goal of phase 2 was to initiate re-education of gait with the walker. General criteria for progressing to the next phase involved the patient following commands, having stable hemodynamic and acceptable oxygen, being capable of transfer to the chair with the assistance of a walker, and walker re-education. ${ }^{27-33}$

\section{Phase 3}

This phase included patients in the acute/subacute phase with multiple medical problems or resolving medical problems and able to participate actively in the therapy. The goal of phase 3 was to initiate independent transfer training with a walker and provide progressive walking re-education. General criteria for progressing to the next phase included the patient following commands, being hemodynamically stable, with acceptable oxygen levels, and with improved tolerance to a progressive walking program. ${ }^{34-42}$

\section{Phase 4}

Patients in the subacute phase, who had been weaned from mechanical ventilation, were able to participate actively. The goal of phase 4 was to promote progressive transfers and walking independence. Assessment of physical therapy was carried out, the phase of the program in which the patient should be included was determined, and the mobility plan of care was established. ${ }^{12,13}$

\section{Data Collection Methods}

A total of 30 patients were selected as participants in the study from BRB Hospitals Ltd., Dhaka. Fifteen patients were included in the ICU treatment group and another 15 were in the intervention group. Graded early mobilization was provided as an intervention to all participants of the intervention group by a professionally qualified physiotherapist for 5 sessions to each participant. Another senior physiotherapist was assigned the role of a data collector for both control and intervention groups to reduce bias and ensure the blind nature of the study.

\section{Data Processing and Analysis}

All interviewed questionnaires were checked for their completeness, accuracy, and consistency to exclude missing or inconsistent data. The data was processed and analyzed using an SPSS 16.0 version software program. Data were cleaned and edited by running frequency and cross-tabulation. Data processing was done by coding, recoding, sorting, categorizing, computing, etc. The tools needed for the study were consent paper, questionnaire, paper, pen, file, calculator, computer, and printer.

\section{Results}

In the control group mean FIM score was 17.40 (SD \pm 4.881$)$ and in the intervention group mean score was 65.70 ( $S D \pm 12.184)$. The mean difference was statistically significant in the ' $t$ ' test. ( $p$-value $>0.001$ ). In the control group, the mean GAD-7 score was 19.50 (SD \pm 2.717 ), and in the intervention group the mean GAD-7 score was 7.5 $(\mathrm{SD} \pm 2.593)$. The mean difference was statistically significant in the ' $t$ ' test ( $p$-value $>0.001$ ) (Table 1 ).

$100 \%$ of participants of the control group were out of bed first at the date of discharge from ICU. But it was possible for a maximum $(90 \%)$ of participants of the intervention group to get out of bed on the second (2nd) day within ICU.

Only $10 \%$ of participants were out of bed on the third day. The mean length of ICU stay in the control group was $5.60(\mathrm{SD} \pm 1.07497)$ and in the intervention group it was 3.10 (SD \pm 0.56765$)$.

The mean difference was statistically significant in the ' $t$ ' test. ( $p$-value $>0.001$ ).In the Chi-square result, it was found there is a significant relationship between participant's 1st day out of bed and FIM improvement and GAD-7 improvement ( $p$-value $>0.001$ ) (Tables 2 and 3). 
Table 1: Different variables in two groups

\begin{tabular}{lll}
\hline Variables & Control group & Intervention group \\
\hline FIM score & Mean score: 17.40 & Mean score: 65.70 \\
& SD: \pm 4.881 & SD: \pm 12.184 \\
& Variance: 23.822 & Variance: 148.456 \\
GAD-7 score & Mean score: 19.50 & Mean score: 5.50 \\
& SD: \pm 2.171 & SD: \pm 2.593 \\
& Variance: 4.711 & Variance: 6.722 \\
Day 1stout of bed & Mean: 5.60 & Mean: 2.10 \\
& SD: \pm 1.075 & SD: \pm 0.316 \\
& Variance: 1.156 & Variance: 0.1 \\
Length of ICU stay & Mean: 5.60 & Mean: 3.10 \\
& SD: \pm 1.075 & SD: \pm 0.568 \\
& Variance: 1.156 & Variance: 0.322 \\
\hline
\end{tabular}

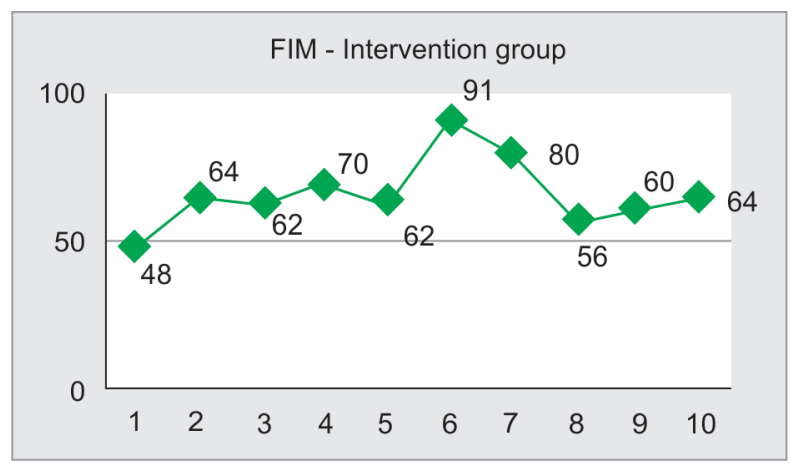

Fig. 1: Functional improvement measure (Intervention Group)

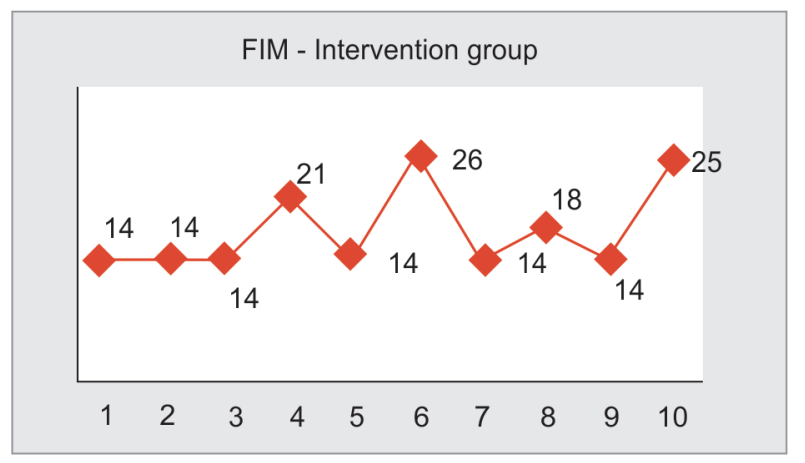

Fig. 2: Functional improvement measure (Control Group)

Table 2: The ' $t$ ' test findings for FIM change

\begin{tabular}{lll}
\hline & Control group & Intervention group \\
\hline Data & $14,14,15,21,14,26,14$, & $60,56,80,91,62,70,62$, \\
& $18,14,25$ & $64,48,64$ \\
Mean & 17.40 & 65.70 \\
SD & 4.81318 & 12.18423 \\
(df) & & 18 \\
Numbers & 10 & 10 \\
' $t$ ' & & 11.635 \\
' $p$ 'value & & Less than 0.001 \\
\hline
\end{tabular}

Table 3: The ' $t$ ' test findings for GAD-7 score change

\begin{tabular}{lll}
\hline & Control group & Intervention group \\
\hline Data & $19,21,20,18,19,14,21,21$, & $6,7,3,3,4,3,4,7,11,7$ \\
& 21,20 & \\
Mean & 19.50 & 5.50 \\
$(\mathrm{SD})$ & 2.17051 & 2.59272 \\
$\mathrm{df}$ & 13 & \\
Numbers & 10 & 10 \\
' $t$ ' & 13 & \\
' $p$ ' value & $<0.001$ & \\
\hline
\end{tabular}

According to the findings of Chi-square, the " $p$ " value was less than 0.001 . So, participants who got out of bed early from the intervention group got better improvement. So, there is a significant relationship between participant's 1st day out of bed and FIM improvement and GAD-7 improvement.

\section{Discussion}

The main objective of this study was to explore the effectiveness of graded early mobilization on psychomotor status and duration of ICU stay of the patients who were mechanically ventilated in the ICU of a hospital setting.

In this study, we have found that graded early mobilization significantly improved patient functional independence. Almost every patient in the control group scored very low in the FIM assessment (mean: 17.40). On the other hand, as all the participants of the intervention group wear treated in ICU with graded mobilization and most of them were brought out of bed on the 2nd day within ICU, they became functionally very active within ICU stay. During discharge from ICU, their FIM assessment score was very high (mean: 65.70) compared to the control group and the change was statistically significant.

In the current study, we observed some psychological issues of participants during ICU stay. In the control group, $80 \%$ of participants were reported with severe anxiety level in GAD-7 assessment, only $20 \%$ were in the moderate anxiety level and no one reported normal during ICU stay (GAD-7 mean: 19.50).

But in the intervention group, the scenario was different. All the participants received graded mobilization in the ICU setting. The professional physiotherapist did plenty of verbal interaction with each participant which may have played a vital role in their psychological status as well. The result showed that only $40 \%$ of participants responded to mild anxiety and $10 \%$ of participants responded to moderate anxiety in GAD-7 assessment. But 50\% of participants responded with an anxiety-free score in GAD-7 assessment. (mean GAD-7: 5.50). In comparison to the control group, the change was statistically significant ( $p$-value was less than 0.001 ).

In this study, findings were in some features similar and some area better. It was found from the statistical analysis from functional improvement measure (FIM) of patients that at $18 \mathrm{df}$ the value of ' $t$ ' at $5 \%$ level of significance was 11.635 as found on reference to ' $t$ ' table (appendix). Thus, the probability of occurrence $(p)$ of the value obtained (11.635) by chance is much less than 0.001 , the critical or $5 \%$ level of significance. ' $p$ ' comes to $<0.001$ on referring to the ' $t$ ' table. It can occur less than 01 times in 1000 which occurs very rarely by chance. So it showed that graded early mobilization is significantly more effective rather than Only ICU Treatment for 
functional improvement and independence of patients. So this study confirms the hypothesis and rejects the null hypothesis.

For reduction of ICU-acquired anxiety, at $13 \mathrm{df}$ the value of ' $t$ ' at $5 \%$ level of significance was 13 as found in reference to the ' $t$ ' table (appendix). Thus, the probability of occurrence $(p)$ of the value obtained (13) by chance is much less than 0.001 , the critical or $5 \%$ level of significance. ' $p$ ' comes to $<0.001$ on referring to the ' $t$ ' table. It can occur less than 01 times 1000 which means very rarely by chance. So it showed that graded early mobilization is significantly more effective rather than only ICU Treatment for the reduction of ICU acquired anxiety. So this experiment establishes the hypothesis and rejects the null hypothesis.

In respect of ICU stay, at $18 \mathrm{df}$ the value of ' $t$ ' at $5 \%$ level of significance is 6.50 as found in reference to the ' $t$ ' table (appendix). Thus, the probability of occurrence $(p)$ of the value obtained (6.50) by chance is much less than 0.001 , the critical or $5 \%$ level of significance. ' $p$ ' comes to $<0.001$ on referring to the ' $t$ ' table. It can occur less than 01 times in 1000 which occurs very rarely by chance. This finding showed that graded early mobilization is significantly more effective rather than Only ICU Treatment for the reduction of length of ICU stay. So this experiment confirmed the hypothesis and rejected the null hypothesis.

In this study, the Chi-square test was calculated to determine any significant relationship between the outcome frequencies and variables in one or more categories. There was no significant relationship between gender and FIM improvement and GAD-7 improvements ( $p=0.245$ ). So, there is some relationship showed that of Graduation and higher education holder participants are better to get improvement but the change was not statistically significant ( $p=0.077)$. But, there was a strong relationship between the participant's 1st day out of bed and improvement. According to the findings of the Chi-square, the $p$-value was less than 0.001 . So, participants from the intervention group who could not get out of bed early improved better. So, there is a significant relationship between participant's 1st day out of bed and FIM improvement and GAD-7 improvement.

\section{Limitation and Recommendation}

Patients in ICU may not be similarly critical and their medical conditions also may not be of a similar level. So, there is a possibility to enroll medically less ill patients in the intervention group and more ill patients in the control group which may affect the findings of this study.

The results of this study have pursued the effects of graded early mobilization for 10 session's intervention effects whereas its long-term effects are unknown so a follow-up research study of these interventions would be more valid.

The study was conducted in one general hospital only. Further research can be recommended with multiple hospitals ICU to get varieties of patients where the chance of comparison study is also feasible.

\section{CONCLUSION}

This study has proven that graded early mobilization is statistically highly significant to gain functional improvement of a patient within ICU stay and reduces the length of ICU stay. Not only functional improvement, but GAD-7 assessment findings were also significantly better during discharge from ICU. So participants of the intervention group got better psychological status and less anxiety level compared with the control group. It was a study with a very small group of respondents with only 05 sessions of intervention. So, it is strongly recommended to conduct a follow-up study to see the long-term effectiveness.

\section{OrCID}

Bijoy Das ๑ https://orcid.org/0000-0002-8733-2176

Sanchita Saha 구 https://orcid.org/0000-0001-9995-2535

Feroz Kabir (1) https://orcid.org/0000-0002-5885-4514

Sazzad Hossain 10 https://orcid.org/0000-0002-8433-2752

\section{References}

1. Aletreby WT, Mumtaz SA, Al Harthy AM, Shahzad SA, Ramadan OE. Outcome of early mobilization of critically ill patients: a propensity score matching trial. Anticoagulation during ECMO: the past, present, and future. J Intensive Crit Care 2018;4(3):13. DOI: 10.21767/2471-8505.100115.

2. Barlow DH. Anxiety and its disorders: the nature and treatment of anxiety and panic. New York: The Guilford Press; 2004.

3. Cindy H, Gayle L, Shelley S. Psychological sequelae following ICU admission at a level 1 academic South African hospital.SAJCC 2010;26(2).

4. Davydow DS, Katon WJ, Zatzick DF. Psychiatric morbidity and functional impairments in survivors of burns, traumatic injuries, and ICU stays for other critical illnesses: a review of the literature. Int Rev Psychiatry 2009;21:531-538. DOI: 10.3109/09540260903343877.

5. De Jonghe B, Sharshar T, Lefaucher JP, Authier FJ, Durand-Zaleski I, Boussarsar M. Paresis acquired in intensive care unit: a prospective multicenter study. JAMA 2002;288(22):2859-2867. DOI: 10.1001/ jama.288.22.2859.

6. DesaiS, LawT,Needham D. Long-term complications of critical care. Crit Care Med 2011;39(2):371-379. DOI: 10.1097/CCM.0b013e3181fd66e5.

7. Diagnostic and statistical manual of mental disorders. Washington: American Psychiatric Association; 1994.

8. Drolet A, De Juilio P, Harkless S, Henricks S, Waters C, Wiliams S. Move to improve: the feasibility of using an early mobility protocol to increase ambulation in the intensive and intermediate care setttings. Phys Ther J 2012;93(2):1-11. DOI: 10.2522/ptj.20110400.

9. Ekiz T, Pazarli AC, Esquinas AM. Early mobilization after mechanical ventilation: a question of details and time. Arch Phys Med Rehabil 2017;98(7):1490. DOI: 10.1016/j.apmr.2017.02.012.

10. Garnacho-Montero J, Amaya-Villar R, Garcia-Garmendia JL, MadrazoOsuna J, Ortiz-Leyba C. Effect of critical illness polyneuropathy on the withdrawal from mechanical ventilation and the length of stay in septic patients. Crit Care Med 2005;33(2):349-354. DOI: 10.1097/01. CCM.0000153521.41848.7E.

11. Gosselink R, Bott J, Johnson M. Physiotherapy for adult patients with critical illness. Recommendation of European Respiratory Society and European Society of Intensive Care Medicine Task force on Physiotherapy for Critically ill patients. Intensive Care Med 2008;34(7):1188-1199. DOI: 10.1007/s00134-008-1026-7.

12. Grosu HB, Lee YI, Lee J, Eden E, Eikermann M, Rose KM. Paresis acquired in intensive care unit: a prospective multicenter study. JAMA 2012;288(22):2859-2867. DOI: 10.1001/jama.288.22.2859.

13. Hodgson C, Bellomo R, Berney S, Bailey M, Buhr H, Denehy L. Early mobilization and recovery in mechanically ventilated patients in the ICU: a binational, multi-centre, prospective cohort study. Crit Care 2015;19(1):1-10. DOI: 10.1186/s13054-015-0765-4.

14. Horta D, Plazas C, Serrano C (1998). The role of The Psychologist in an Intensive Care Unit. Faculty of Psychology PUJ. Unpublished document. [Taken on 02/10/2020]: from: http://www.scielo.org.co/ scielo.php?script=sci_arttext\&pid=S1657-92672006000300014.

15. Jones C, Griffiths RD, Humphris G, Skirrow PM. Memory, delusions, and the development of acute posttraumatic stress disorder-related symptoms after intensive care. Crit Care Med 2001;29(3):573-580. DOI: 10.1097/00003246-200103000-00019. 
16. Kress JP, Hall JB. Risk factors among ICU patient and their musculoskeletal features. J Intensive Crit Care 2014;11(3):13. DOI: 10.21567/2471-855.101515.

17. Kress JP, Hall JB. ICU-acquired weakness and recovery from critical illness. N Engl J Med 2014;370:1626-1635. DOI: 10.1056/ NEJMra1209390.

18. Levine S, Nguyen T. Early intensive care unit mobility therapy in the treatment of acute respiratory failure. Crit Care Med 2008;36:22382243. DOI: 10.1097/CCM.0b013e318180b90e.

19. Lipshutz A, Gropper M. Aquired neuromuscular weakness and early mobilization in the intensive care unit. Anesthesiology 2013;118(1):202-214. DOI: 10.1097/ALN.0b013e31826be693.

20. McCarren B, Alison JA, Herbert RD. Manual vibration increases expiratory flow rate via increased intrapleural pressure in healthy adults: an experimental study. Aust J Physiothery 2015;52:267-271. DOI:10.1016/S0004-9514(06)70006-x.

21. Meesen RL, Dendale P, Cuypers K. Neuromuscular electrical stimulation as a possible means to prevent muscle tissue wasting in artificially ventilated and sedated patients in the intensive care unit: a pilot study. Neuromodulation 2010;13(4):315-320. DOI:10.1111/j.15251403.2010.00294.x.

22. Meng Y, Zhan-Ying Ma, Meng-Jie L, Chu-Yun C, Yi Jin. Early mobilization for mechanically ventilated patients in the intensive care unit: a systematic review and meta-analysis. Front Nurs 2018;5(4). DOI: 10.1515/fon-2018-0039.

23. Morris PE. Moving our critically ill patients: mobility barriers and benefits. Crit Care Clin 2007;23(1):1-20. DOI: 10.1016/j.ccc.2006. 11.003.

24. Morris PE, Goad A, Thompson C, Taylor K, Harry B, Passmore L. Early intensive care unit mobility therapy in the treatment of acute respiratory failure. Crit Care Med 2008;36(8):2238-2243. DOI: 10.1097/ CCM.0b013e318180b90e.

25. Morris PE, Griffin L, Berry M. Receiving early mobility during an intensive care unit admission is a predictor of improved outcomes in acute respiratory failure. Am J Med Sci 2011;341(5):373-377. DOI: 10.1097/MAJ.0b013e31820ab4f6.

26. Myers DG. Therapy. Psychology. 9th Ed. New York: Worth Publishers; 2010.

27. Narasimman S, Mohammad H. Early mobilization in ICU. Current perspective and future directions. Physio Times 2010;2:18-23.

28. Nyland BA, Spilman SK, Halub ME, Lamb KD, Jackson JA, Oetting TW. A preventative respiratory protocol to identify trauma subjects at risk for respiratory compromise on a general in-patient ward. Respir Care 2016;61(12):1580-157. DOI: 10.4187/respcare.04729.

29. Patman S, Sanderson D, Blackmore M. Physiotherapy following cardiac surgery: is it necessary during the intubation period? Aust J Physiother 2001:;47(1):7-16.DOI: 10.1016/S0004-9514(14)60294-4.
30. Pohlman MC, Schweickert WD, PohIman AS. Feasibility of physical and occupational therapy beginning from initiation of mechanical ventilation. Crit Care Med 2010;38(11):2089-2094. DOI: 10.1097/ cCM.0b013e3181f270c3.

31. Poulsen JB, Møller K, Jensen CV, Weisdorf S, Kehlet H, Perner A. Effect of transcutaneous electrical muscle stimulation on muscle volume in patients with septic shock. Crit Care Med 2011;39(3):456-461. DOI: 10.1097/CCM.0b013e318205c7bc.

32. Priyakshi B, Narasimman S, D'silva C, Shabari K. Effect of graded early mobilization versus routine physiotherapy on the length of intensive care unit stay in mechanically ventilated patients: a randomized controlled study. Int J Health Allied Sci 2012;1(3):172-177. DOI: 10.4103/2278-344X.105081.

33. Puthucheary ZA, Rawal J, McPhail M, Connolly B, Ratnayake G, Chan $P$, et al. Acute skeletal muscle wasting in critical illness. JAMA 2013;310(15):1591-1600. DOI:10.1001/jama.2013.278481.

34. Rodriguez PO, Setten M, Maskin LP. Muscle weakness in septic patients requiring mechanical ventilation: protective effect of transcutaneous neuromuscular electrical stimulation. J Crit Care 2012;27(3):319.e1-319.e8. DOI: 10.1016/j.jcrc.2011.04.010.

35. Sareen J, Cox BJ, Afifi TO, de Graaf R, Asmundson GJ, et al. Anxiety disorders and risk for suicidal ideation and suicide attempts: a population-based longitudinal study of adults. Arch Gen Psychiatry 2005;62(11):1249-1257. DOI: 10.1001/archpsyc.62.11.1249.

36. Sommers J, Engelbert RH, Dettling-Ihnenfeldt D, Gosselink R, Spronk $\mathrm{PE}$, Nollet F. Physiotherapy in the intensive care unit: evidencebased, expert-driven, practical statement and rehabilitation recommendations. Clin Rehabil 2015;29(11):1051-1063. DOI: 10.1177/ 0269215514567156.

37. Stiller K. Safety issues that should be considered when mobilizing critically ill patients. Crit Care Clin 2007;23(1):35-53. DOI: 10.1016/j. ccc.2006.11.005.

38. Turner JM, Smith PC, Ramchandani C, Begen FM, Padkin A. The acute psychobiological impact of the intensive care experience on relatives. Psychol Health Med 2016;21(1):20-26. DOI: 10.1080/ 13548506.2014.997763.

39. Van Der Kolk BA, McFarlane AC, Weisaeth L. Traumatic stress: the effects of overwhelming experience on mind, body and society. New York: Guilford Publications; 2006.

40. Zigmond AS, Snaith RP. The hospital anxiety and depression scale. Acta Psychiatr Scand 1983;67(6):361-370.DOI: 10.1111/j.16000447.1983.tb09716.x.

41. Sharon AS. Mechanical Ventilation Learning Pakage, CNC, Liverpool ICU, SWSLHD; 2016. pp. 16-38.

42. Mahajan BK. Methods of biostatistics for medical students and research workers. Jaypee Brothers medical publishers ( $p$ ) limited; 2005. pp. 130-156. 